\title{
r

\section{Clinical and radiological results after coracoclavicular ligament reconstruction for type III acromioclavicular joint dislocation using three different techniques. A retrospective study}

\author{
ALBERTO VASCELLARI ${ }^{1}$, STEFANO SCHIAVETTI ${ }^{1}$, GIUSEPPE BATTISTELLA ${ }^{2}$ \\ ENRICO REBUZZI ${ }^{1}$, NICOLÒ COLETTI ${ }^{1}$ \\ 1 Orthopaedic and Traumatology Department, Oderzo Hospital, Oderzo, Italy \\ 2 Treviso Hospital, Treviso, Italy
}

\begin{abstract}
Purpose: the purpose of this retrospective study was to present the outcomes of three different techniques for the treatment of type III acromioclavicular joint dislocations: arthroscopic TightRope (TR), arthroscopic GraftRope (GR), and open reconstruction of the coracoclavicular (CC) ligament using the Ligament Augmentation and Reconstruction System (LARS). Methods: eighteen patients underwent clinical and radiological evaluations after a mean follow-up time of 43 months. The following clinical outcome measures were considered: the Disability of the Arm, Shoulder and Hand outcome measure (DASH), the Nottingham Clavicle Score (NCS), and the Constant score (CS). On X-rays, the CC distance was measured. Results: the median DASH score at follow-up was 12.5 in the TR group, 5 in the GR group, and 4.2 in the LARS group. The median NCS value was 88 in the TR group, 88 in the GR group, and 91 in the LARS group. The median CS was 100 in the TR group, 95 in the GR group, and 94.5 in the LARS group. The mean CC distance was $10.3 \mathrm{~mm}$ in the TR group, 13.8 in the GR group, and 16.6 in the LARS group. Conclusions: all three techniques proved to be reliable in providing good clinical outcomes, although none of the studied techniques demonstrated reliability in maintaining anatomical reduction after surgery. Level of evidence: Level III, retrospective comparative study.
\end{abstract}

\footnotetext{
Corresponding Author:

Alberto Vascellari, MD

Orthopaedic and Traumatology Department, Oderzo Hospital

Via Luzzatti 33, 31046 Oderzo, Italy

E-mail:mascvoz@gmail.com
}

Key Words: acromioclavicular dislocation, coracoclavicular reconstruction, GraftRope, LARS, artificial ligament, TightRope.

\section{Introduction}

Despite their frequent occurrence, there is still much debate over the management of acromioclavicular joint (ACJ) dislocations. Rockwood type III injuries are the most controversial questions having been raised about whether these should be treated surgically or not (1). Although advocates of non-surgical treatment suggest that patients often demonstrate excellent clinical results and regain painless shoulder function, many Authors advocate a surgical approach, especially in young, active individuals and manual workers, due to the potential risks, otherwise, of shoulder deformity and poor function due to chronic instability and pain $(2,3)$.

Studies comparing the results of surgical and conservative treatment of type III ACJ separations show that surgical intervention provides no substantial benefit, but criticism of these studies' conclusions focused on the fact that the ACJ fixation was performed without coracoclavicular (CC) ligament reconstruction $(2,3)$. Given that the CC ligaments are considered the primary suspensory restraint of the ACJ against superior and posterior translation of the distal clavicle with respect to the scapula $(4,5)$, current techniques focus on anatomical restoration of the CC ligament complex $(6,7)$.

The LARS (Ligament Augmentation and Reconstruction System) (Corin, Cirencester, UK) has proven to provide highly resistant, separate reconstruction of the conoid and trapezoid ligaments; this is achieved through the use of biomimetic artificial ligament made of polyester fibers (8-10). 
The arthroscopically-assisted TightRope (TR) (Arthrex, Naples, FL, USA) technique is a minimally invasive method used to stabilize the ACJ and augment the CC complex with a high-strength suture (11-13). The GraftRope (GR) (Arthrex), an evolution of the TR technique, combines fixation and stability of the ACJ with the use of a soft-tissue graft to promote biological healing in chronic lesions (14).

The aim of this study was to retrospectively evaluate the mid-term follow-up results of type III ACJ dislocations treated using these three different techniques: arthroscopic TightRope, arthroscopic GraftRope and open CC ligament reconstruction using LARS. The hypothesis of the study was that the difference in outcome between the three techniques was non-significant.

\section{Methods}

From 2005 to 2008, the standard treatment for type III ACJ dislocations at our institution was open LARS CC ligament reconstruction. In 2008 the arthroscopicallyassisted TR technique was introduced in order to minimize surgical trauma and improve the postsurgical cosmetic appearance. The GR, an evolution of the TR technique, was subsequently introduced to combine high-strength-suture temporary fixation and a biological graft within the construct for long-term ligament incorporation, and was also proposed for acute injuries. The use of these three techniques resulted in the three patient populations analyzed in this retrospective study.

\section{Study population}

All patient charts relating to isolated type III ACJ dislocation operations carried out at our institution between February 2005 and December 2011 were retrospectively reviewed. All the patients underwent preoperative anteroposterior and axillary shoulder radiographs, and were classified as Rockwood III by the surgeon. An ACJ injury that was older than one month was defined chronic. Candidates for surgery included younger patients who regularly performed heavy manual work, highly active patients and athletes, patients with unacceptable cosmetic deformities or in unacceptable pain, as well as patients with functional disabilities following conservative treatment.

Exclusion criteria were: immature bone, open dislocation, neurovascular injury, clinical, radiological or arth- roscopic diagnosis of associated injuries of the rotator cuff, glenoid labrum or articular cartilage of the glenohumeral joint, or biceps tendon pathologies, and patients who had previously undergone shoulder surgery, on their injured or uninjured shoulder.

Of the 22 patients selected, 6 were operated on using the arthroscopic TR technique, 6 with arthroscopic GR technique, and 10 with open LARS CC ligament reconstruction. The patients were divided into three groups accordingly: TR group, GR group and LARS group. These 22 patients were asked to participate in the study, which involved new clinical as well as radiological examinations. Four patients $(1$ treated with the TR technique, 1 with the GR technique, and 2 with open LARS CC ligament reconstruction) were not contactable or declined to return for evaluation. Thus, 18 patients were re-evaluated: 5 from the TR group, 5 from the GR group, and 8 from the LARS group. Three patients declined new radiological follow-up evaluations, even though they had undergone the clinical follow-up examination; 2 of these patients provided recent standard radiographs. The patient data are shown in Table 1.

The study protocol was approved by the local ethics committee, and the study was performed in accordance with the ethical standards of the 1964 Declaration of Helsinki as revised in 2000. It also complied with recently published ethical standards in clinical and field science research (15). All the subjects were informed of the purpose and procedure of the study, and of any known risks, and all gave their informed consent to participate in it.

\section{Interventions}

The arthroscopic technique was performed through three standard arthroscopic portals (posterior, anterior and anterolateral). The coracoid undersurface was circumferentially prepared using a mechanical shaver and a radiofrequency device. A drill guide was inserted through the anterior portal and positioned under the base of the coracoid as close to the scapula as possible. A $1.5 \mathrm{~cm}$ incision was made perpendicular to the clavicle, approximately $2.5 \mathrm{~cm}$ medial to the lateral border of the clavicle, and a guide pin was inserted and advanced through the clavicle and coracoid. The tip of the guide pin could be seen under the base of the coracoid, thus making it possible to check the position of the pin in relation to the anterior and posterior cortices of the 
Table 1. Patient data.

\begin{tabular}{|c|c|c|c|c|c|c|c|}
\hline Patient No. & Gender & Age (y) & $\begin{array}{l}\text { Follow-up } \\
\text { (mo) }\end{array}$ & $\begin{array}{l}\text { Injury } \\
\text { mechanism }\end{array}$ & Side & Surgery & $\begin{array}{l}\text { Time I-R } \\
\text { (days) }\end{array}$ \\
\hline 1 & $\mathrm{M}$ & 48 & 88 & Fall & $\mathrm{R}$ & LARS & 143 \\
\hline 2 & $\mathrm{M}$ & 48 & 86 & Biking accident & $\mathrm{R}$ & LARS & 13 \\
\hline 3 & $\mathrm{M}$ & 58 & 64 & Fall & $\mathrm{R}$ & LARS & 4 \\
\hline 4 & $\mathrm{M}$ & 51 & 62 & Fall & $\mathrm{R}$ & LARS & 360 \\
\hline 5 & $\mathrm{~F}$ & 37 & 53 & Car accident & $\mathrm{R}$ & TR & 17 \\
\hline 6 & $\mathrm{M}$ & 31 & 51 & Motorcycling accident & $\mathrm{R}$ & TR & 6 \\
\hline 7 & $\mathrm{M}$ & 31 & 51 & Skiing accident & $\mathrm{L}$ & TR & 12 \\
\hline 8 & $\mathrm{M}$ & 62 & 48 & Car accident & $\mathrm{L}$ & TR & 300 \\
\hline 9 & $\mathrm{M}$ & 46 & 40 & Motorcycling accident & $\mathrm{R}$ & GR & 270 \\
\hline 10 & $\mathrm{M}$ & 42 & 38 & Biking accident & $\mathrm{R}$ & GR & 15 \\
\hline 11 & $\mathrm{M}$ & 41 & 36 & Biking accident & $\mathrm{R}$ & GR & 10 \\
\hline 12 & $\mathrm{M}$ & 64 & 35 & Fall & $\mathrm{L}$ & LARS & 11 \\
\hline 13 & $\mathrm{M}$ & 47 & 31 & Fall & $\mathrm{R}$ & GR & 10 \\
\hline 14 & $\mathrm{M}$ & 29 & 30 & Motorcycling accident & $\mathrm{L}$ & GR & 9 \\
\hline 15 & $\mathrm{~F}$ & 52 & 23 & Car accident & $\mathrm{L}$ & LARS & 420 \\
\hline 16 & $\mathrm{M}$ & 67 & 20 & Biking accident & $\mathrm{L}$ & TR & 10 \\
\hline 17 & $\mathrm{M}$ & 29 & 15 & Skiing accident & $\mathrm{R}$ & LARS & 10 \\
\hline 18 & M & 57 & 12 & Biking accident & $\mathrm{R}$ & LARS & 296 \\
\hline
\end{tabular}

Abbreviations: Time I-R, time between injury and reconstruction surgery; TR, TightRope; GR, GraftRope; LARS, Ligament Augmentation and Reconstruction System.

coracoid. A $4 \mathrm{~mm}$ cannulated drill was advanced over the pin and through the clavicle and coracoid, and left in situ in order to advance a Nitinol passing wire that was then pulled out of the anterior portal using an arthroscopic grasper. The traction sutures of the TR were retrieved from the anterior portal using the suture passing wire. The oblong button of the TR was then, under observation, advanced through the clavicle and the coracoid until it exited the coracoid; it was then flipped onto the underside of the coracoid base. Once the oblong button was securely in place, the clavicle was manually reduced, the round button of the TR was moved down to the surface of the clavicle, and the sutures were tied over the top of the TR.

The GR evolution of the TR technique is designed to accept autograft or allograft tissue, such as semitendinosus, gracilis or tibialis anterior tendon soft-tissue grafts tied over the oblong button of the GR before surgery. Similarly to the TR technique, a $6 \mathrm{~mm}$ bone tunnel was drilled over a guide pin through the clavicle and coracoid, and then the GR was advanced through the clavicle and the coracoid while pulling a traction suture. The round button was advanced down to the surface of the clavicle and the sutures were tied over the top of the GR, while the clavicle was manually reduced. A $5.5 \mathrm{~mm}$ screw was then placed as an interference screw between the tensioned graft limbs for final construct fixation.

In the LARS technique, a 3- to $5 \mathrm{~cm}$ incision was made in the sagittal plane, extending up from the coracoid over the clavicle. After identification and exposure of the coracoid process, two transosseous tunnels were made in the lateral clavicle, one on each side of the coracoid. Great care was taken to make these tunnels oblique, so as not to weaken the clavicle by damaging the anterior and posterior cortices: the lateral tunnel runs forward at an oblique angle and the medial runs backward at an oblique angle. A hook-like guide instrument provided by the LARS company, with a loaded wire loop, was passed medially under the coracoid from medial to lateral and was passed around the coracoid. The ligament was then loaded in the loop and passed under the coracoid. Wire loops were passed through the clavicular tunnels, and the ligament ends were fixed to the bone tunnels with titanium interference screws. During the study period, the patients were treated by four different senior orthopedic surgeons.

The patients in all groups kept their treated arm in a sling for two weeks, and allowed a limited range of motion; they were then allowed to use their arm for 
daily activities and below-shoulder-level movements for up to four weeks post-operation, after which they were allowed to move freely.

\section{Outcome measurements}

A blind observer reviewed all 18 patients, both clinically and radiographically. The subjective Disability of the Arm, Shoulder and Hand outcome measure (DASH) (16), the Nottingham Clavicle Score (NCS) (17), and the Constant Score (CS) (18) were used to clinically evaluate the ACJ.

The new bilateral radiographs taken at the time of the re-examination consisted of anteroposterior ACJ and axillary shoulder radiographs, as well as stress radiographs performed using $5 \mathrm{~kg}$ weights suspended by wrist straps from each arm. The degree of displacement of the ACJ was evaluated by measuring the $\mathrm{CC}$ distance on the anteroposterior view (for vertical displacement) and on the axillary view (for horizontal displacement). According to Bearden et al. (19), the finding of a CC distance 25 to $50 \%$ greater than that of the normal side indicates complete CC ligament disruption; accordingly a CC distance on the affected side found to be more than $25 \%$ greater than that of the normal side was considered a radiological failure. Osteoarthritis (OA), ossifications between the clavicle and the coracoid, and clavicular osteolysis were evaluated on anteroposterior stress views taken at the time of the re-examination, or on standard anteroposterior views in patients who had refused new radiographic follow-up evaluations but provided recent standard radiographs. Shoulders were considered normal when there were no signs of OA. Cases with mild OA showed a narrowing of the joint space and subchondral sclerosis; those with moderate OA showed subchondral sclerosis and osteophytes, while cases with severe $\mathrm{OA}$ had severe joint deformation. Ossification of the CC ligaments was categorized into three degrees: mild in the presence of thin calcifications around the CC ligaments, moderate if there were large amounts of radiopaque material around the CC ligaments, and severe in cases of complete calcification of the CC ligaments. Three degrees of clavicular osteolysis were also identified. Osteolysis was considered mild if the clavicular end was poorly defined or showed scattered areas of demineralization, moderate if there was evident demineralization, yet extending to less than half of the clavicular extremity, and severe in the presence of clearly visible demineralization affecting more than half of the clavicular extremity. Implant migration was also evaluated in the arthroscopic groups. Migration either on the clavicular or on the coracoid side was classified as: i) none, ii) migration to the level of the superior cortex (only the clavicular side), iii) migration into the clavicle or coracoid, or iv) complete dislocation.

\section{Data analysis}

Statistical analysis was performed using SPSS software, version 13.0; (SPSS Inc, Chicago, IL) and WinPepi (version 11.9). Statistical significance was set at $\mathrm{p}<0.05$. The DASH results, NCS results and radiological outcomes were correlated using the Pearson correlation coefficient. The same parameters were correlated with the presence of ossification, $\mathrm{OA}$, osteolysis and implant migration using the Kendall's tau b rank correlation coefficient. The results are described using mean values and standard deviation (SD) for parametric values and median values (interquartile range, range) for nonparametric ones.

\section{Results}

The male:female ratio was 16:2. At the time of surgery, the patients' mean age was 45.6 years (SD 17.5 years) in the TR group, 41 years (SD 7.2 years) in the GR group, and 50.9 years (SD 10.4 years) in the LARS group. The acute:chronic disease ratio was 4:1 in the TR group, 4:1 in the GR group, and 4:4 in the LARS group. The mean follow-up time was 46 months in the TR group, 41 months in the GR group, and 48 months in the LARS group. All the outcomes are reported in Table 2.

The median DASH score at follow-up was $12.5\left(1^{\text {st }}\right.$ 3rd quartile 4.2-8.35, range 2.5-17.5) in the TR group, 5 ( $1^{\text {st }}-3$ rd quartile 2.5-7.9, range 1.7-13.3) in the GR group, and 4.2 ( $1^{\text {st }}-3$ rd quartile $1.5-12.7$, range $\left.0-45\right)$ in the LARS group.

The median NCS score was 88 (1 ${ }^{\text {st }}-3$ rd quartile $80-93$, range $76-96)$ in the TR group, 88 (1 $1^{\text {st }}-3$ rd quartile 84 91, range 80-94) in the GR group, and 91 ( $1^{\text {st }}-3$ rd quartile 81.5-95.5, range 52-100) in the LARS group. The median CS was 100 ( $1^{\text {st }}-3$ rd quartile 100-100, range 93-100) in the TR group, 95 (1 $1^{\text {st }}-3$ rd quartile 89 100 , range $88-100)$ in the GR group, and 94.5 ( $1^{\text {st }}-3$ rd quartile 92.25-97, range 78-100) in the LARS group. 
Table 2. Clinical and radiological results.

\begin{tabular}{|c|c|c|c|c|c|c|c|c|c|c|c|c|}
\hline $\begin{array}{l}\text { Patient } \\
\text { No. }\end{array}$ & Surgery & DASH & $\begin{array}{l}\text { Pain } \\
\text { ACJ }\end{array}$ & NCS & $\mathrm{CS}$ & $\begin{array}{l}\text { CC Dist } \\
\text { (Stress) }\end{array}$ & $\begin{array}{l}\text { CC Dist } \\
\text { (Stress)Ctrl }\end{array}$ & Arthritis & Ossification & Lysis & $\begin{array}{l}\text { Button } \\
\text { migration }\end{array}$ & $\begin{array}{l}\text { Button } \\
\text { type }\end{array}$ \\
\hline 1 & LARS & 4.2 & $\mathrm{~N}$ & 90 & 93 & & & & & & & \\
\hline 2 & LARS & 1.7 & $\mathrm{~N}$ & 100 & 90 & 14.1 & 10.6 & moderate & moderate & none & & \\
\hline 3 & LARS & 35.8 & $\mathrm{~N}$ & 56 & 96 & 13.6 & 11.4 & mild & moderate & mild & & \\
\hline 4 & LARS & 4.2 & $\mathrm{~N}$ & 90 & 94 & 12.8 & 4.9 & none & none & severe & & \\
\hline 5 & TR & 17.5 & $\mathrm{~N}$ & 80 & 100 & 7.3 & 9.8 & none & none & none & into & old \\
\hline 6 & TR & 16.7 & $\mathrm{~N}$ & 76 & 93 & 14 & 10.8 & none & none & none & into & old \\
\hline 7 & TR & 2.5 & $\mathrm{~N}$ & 96 & 100 & & & none & mild & none & into & old \\
\hline 8 & TR & 4.2 & $\mathrm{~N}$ & 88 & 100 & & & mild & none & none & none & old \\
\hline 9 & GR & 2.5 & $\mathrm{~N}$ & 94 & 88 & 18.1 & 11.1 & none & none & none & none & new \\
\hline 10 & GR & 13.3 & $\mathrm{~N}$ & 80 & 95 & 11.1 & 5.5 & none & none & none & none & new \\
\hline 11 & GR & 1.7 & $\mathrm{~N}$ & 94 & 100 & 16 & 13 & none & none & none & none & new \\
\hline 12 & LARS & 0.9 & $\mathrm{~N}$ & 94 & 95 & 17.1 & 8 & mild & moderate & none & & \\
\hline 13 & GR & 10.8 & $\mathrm{~N}$ & 88 & 89 & 12 & 8.4 & moderate & none & none & none & old \\
\hline 14 & GR & 5 & $\mathrm{~N}$ & 84 & 100 & 12.2 & 9.7 & moderate & severe & none & none & new \\
\hline 15 & LARS & 5 & $\mathrm{~N}$ & 92 & 100 & 25.6 & 9.5 & none & none & severe & & \\
\hline 16 & TR & 12.5 & $\mathrm{~N}$ & 90 & 100 & 9.7 & 7.3 & none & none & none & none & new \\
\hline 17 & LARS & 0 & $\mathrm{~N}$ & 100 & 100 & 14.6 & 11.2 & none & none & none & & \\
\hline 18 & LARS & 45 & $\mathrm{~S}$ & 52 & 78 & 18 & 11.5 & none & none & none & & \\
\hline
\end{tabular}

Abbreviations: DASH, Disability of the Arm, Shoulder and Hand outcome measure; CS, Constant score; Pain ACJ, acromioclavicular joint pain; NCS, Nottingham Clavicle Score; CC Dist, coracoclavicular distance (in mm); Stress, stress view; Ctrl, contralateral; LARS, Ligament Augmentation and Reconstruction System; TR, TightRope; GR, GraftRope.

The DASH score was strongly inversely correlated with the NCS score $(p<0.001, r=-.964)$. The ACJ was tender to palpation in one patient belonging to the LARS group, and in none of TR or GR group patients. Three patients in the GR group reported mild tenderness over the superior buttons and suture material, and one of them required button and suture knot removal. Patients who experienced tenderness to palpation over the superior buttons and sutures did not score worse when evaluated using the subjective and functional scoring systems.

In the TR group, the mean CC distance with $5 \mathrm{~kg}$ weight stress loading was $10.3 \mathrm{~mm}$ (SD $3.4 \mathrm{~mm}$ ) on the injured side compared with $9.3 \mathrm{~mm}$ (SD $1.8 \mathrm{~mm}$ ) on the non-injured side. No anteroposterior translation was seen on the axial view. In the GR group, the mean CC distance with $5 \mathrm{~kg}$-weight stress loading was $13.8 \mathrm{~mm}$ (SD $3.0 \mathrm{~mm}$ ), compared with $9.5 \mathrm{~mm}$ (SD $2.8 \mathrm{~mm}$ ) on the non-injured side. There was no anteroposterior translation on the axial view. In the LARS group, the mean CC distance was $16.6 \mathrm{~mm}$ (SD 4.4 $\mathrm{mm}$ ), compared with $9.6 \mathrm{~mm}$ (SD $2.4 \mathrm{~mm}$ ) on the unaffected side. Again, there was no anteroposterior translation on the axial view.
The mean CC difference, which represents the absolute difference in displacement between the injured and non-injured sides, was $2.78 \mathrm{~mm}$ (SD $0.54 \mathrm{~mm}$ ) in the TR group, $4.31 \mathrm{~mm}$ (SD $1.91 \mathrm{~mm}$ ) in the GR group, and $6.96 \mathrm{~mm}(\mathrm{SD} 4.78) \mathrm{mm}$ in the LARS group. The CC distance on the affected side was found to be more than $25 \%$ greater than that of the normal side in six TR+GR group patients and six LARS group patients; thus, the pass:fail ratio, measured in accordance with Bearden et al. (19), was 1:2 in the TR group, 1:4 in the GR group, and 1:6 in the LARS group.

No statistically significant correlation was observed between the clinical scores and the CC distances and CC differences (Tab. 3). The clinical scores, CC distances and CC differences were not correlated with patient age at the time of surgery. The time elapsing between injury and the index procedure was correlated with both CC distance (Pearson correlation $=0.678, p=0.010)$ and $\mathrm{CC}$ difference (Pearson correlation $=-0.763, \mathrm{p}=0.004$ ).

Five patients showed ossifications between the clavicle and the coracoids ( 3 in the LARS group, 1 in the TR group, and 1 in the GR group). Six patients showed 
Table 3. Statistical correlation between clinical scores, CC distance and CC difference.

\begin{tabular}{llll}
\hline \multirow{3}{*}{ CC Dist } & & DASH & NCS \\
& Pearson correlation & -0.095 & 0.083 \\
& Sig. (2-tailed) & 0.735 & 0.768 \\
CC Dist CTRL & N & 15 & 15 \\
& Pearson correlation & 0.193 & -0.169 \\
DD & Sig. (2-tailed) & 0.490 & 0.547 \\
& N & 15 & 15 \\
& Pearson correlation & -0.164 & 0.154 \\
& Sig. (2-tailed) & 0.575 & 0.599 \\
& N & 14 & 14
\end{tabular}

Abbreviations: DASH, Disability of the Arm, Shoulder and Hand outcome measure; NCS, Nottingham Clavicle Score; CC Dist, coracoclavicular distance (in $\mathrm{mm}$ ); CTRL, contralateral; DD, absolute difference in coracoclavicular distance (in mm) between the injured and non-injured sides.

signs of OA ( 3 belonging to the LARS group, 1 to the TR group, and 2 to the GR group). Three patients showed clavicular osteolysis (all 3 belonging to the LARS group).

Three TR group patients showed implant migration into the clavicle. No correlation was found between ossification, OA, osteolysis and implant migration and CC distance or difference.

No patients with ossification, OA, osteolysis and implant migration recorded inferior scoring system scores.

\section{Discussion}

The most important finding of the present study was that all the methods used resulted in high levels of clinical functional outcome and patient satisfaction, in accordance with the literature, in which several different methods of treating ACJ dislocations have been reported to restore shoulder function $(11,20)$.

Another important finding was the high rate of radiological failure registered in this series. Maintenance of anatomical reduction did not seem to be a prerequisite for regaining adequate shoulder function. Boström Windhamre (21) reported a trend whereby patients with recurrent dislocation of $25-100 \%$ of the clavicle width had a better clinical outcome than patients with less than $25 \%$ or more than $100 \%$ recurrent dislocation. Other authors reported that partial loss of reduction did not appear to influence the overall result (20). Boström Windhamre suggested that even an elongated reconstructed ligament improves the stability of the clavicle sufficiently to relieve symptoms and improve shoulder function (21). Therefore, more anatomical healing may be important only to the cosmetic outcome. On the other hand, overcorrection, advocated in some cases in order to anticipate future loss of reduction, can be responsible for brachial plexus compression and should not be an objective of the procedure (22). There was one case in this series in which overcorrection of the CC distance was carried out at the final follow-up stage, and this was associated with an unsatisfactory clinical and functional outcome.

The correlation between injury-surgery interval and radiological outcome recorded in this series suggests that early surgical intervention may be beneficial. Even though, due to a lack of research, an optimal point in time for surgical intervention in ACJ dislocations has yet to be established (23), early treatment of acute ACJ dislocation has proven to provide good clinical results independently of the surgical method (2, $13)$; in fact, acute reduction of the ACJ is easier when surgery is performed within 2-3 weeks of the injury occurring (13).

It is recommended that the TR procedure (a technique in which a prosthetic device is essentially used to maintain ACJ reduction) be performed within one month of the injury (11), when the soft tissues surrounding the clavicle and the coracoid still have sufficient healing potential. In one chronic case in the present series (patient no. 8), the TR procedure was performed and, interestingly, resulted in satisfactory clinical and radiological outcomes. One of the questions that might arise is whether the TR system is capable of providing long-term stability. Walz et al. (24) reported 
two cases with biological reaction in which there were tissue complexes surrounding the TR devices on second-look arthroscopy. Moreover, calcifications along the TR, as observed on postoperative radiographs, might improve scarring of the remaining stumps together with soft-tissue complexes.

The arthroscopically-assisted TR and GR techniques represent minimally invasive methods for augmenting, or non-anatomically reconstructing, a single CC ligament, respectively (11-14). Conversely, LARS anatomical reconstruction provides separate reconstruction of the conoid and trapezoid ligaments. Tauber et al. (20) and Fraschini et al. (8) demonstrated the clinical superiority of anatomical CC reconstruction for ACJ dislocation when compared with non-anatomical approaches. Recent literature suggests that true anatomical reconstruction, attempting to recreate the conoid and trapezoid ligaments, may be advantageous in preventing both recurrent subluxation and anteriorposterior instability $(8,20,23,25)$. That said, for the less experienced arthroscopist, open CC reconstruction is a reliable technique for obtaining highly resistant, separate reconstruction of the conoid and trapezoid ligaments.

The surgical techniques presented have some shortcomings of their own. They require bicortical holes in the clavicle and in the coracoid, which may predispose these structures to fracture. From a biomechanical standpoint, since stabilization in the arthroscopic group is obtained through a single clavicular tunnel, the clavicle is overloaded in a single point, thus increasing the risk of bone erosion and subsequent fracture or migration of implants into the clavicle, as reported by Scheibel et al. (26) and Walz et al. (24); indeed, this is a potential disadvantage of the arthroscopic techniques. Conversely, with LARS, the device is passed through two drill holes in the clavicle and fixed with two interference screws, distributing the load over two points. Therefore, transection of the clavicle due to bone erosion is unlikely to occur.

Patients belonging to the arthroscopic groups who experienced tenderness to palpation over the superior button and sutures did not show worse clinical scores. However, if ongoing problems with the superior implants were reported, for example, if patients were unable to carry a bag with a shoulder strap, we advised them to have the button and suture knots removed after an interval of at least 4 to 6 months from the operation. This proved necessary in only one patient included in this study.

Three patients treated arthroscopically, who showed no history of postoperative trauma presented migration of the superior flip button into the clavicle secondary to penetration of the superior cortex that never progressed beyond the upper third of the collarbone. Clavicular implant migration did not lead to inferior clinical results. Nevertheless, implant migration was regarded as a potential problem because of weakening of the bone and the possibility of stress fractures or fractures occurring after a secondary trauma. It is for this reason that, in 2009, we switched to the second-generation TR system with its larger, round clavicular button. This larger superior button, allowing better load distribution on the superior cortex of the clavicle, prevented migration of the implant in the patients treated with the second-generation TR system (26). Finally, the strong correlation found between the newly developed NCS (17) and the DASH score supports the validity of the NCS for the assessment of ACJ injuries.

This study has a number of important limitations that need to be considered. Its main weakness is that the value of the statistical analysis may be limited by small number of patients involved in the study, which is due to the fact that we considered only highly active patients, manual workers or patients who required surgery for cosmetic reasons. Furthermore, given that this was a retrospective study, the choice of implant was at the surgeons' and patients' discretion. While each patient was allowed to choose a particular technique, it is possible that the surgeons' preferences or the patients' desire for less invasive procedures could have been a factor in the discussion and decision-making process. Thus, aside from the similar injury types and injury mechanisms, the three groups were not perfectly matched; also, there was no pre-selection for treatment. We are also aware of the differences in mean age between the groups of patients studied, albeit these differences were not statistically significant. Lastly, four different surgeons operated on the patients treated for ACJ dislocation.

In conclusion, anatomical CC ligament reconstruction with artificial ligaments, arthroscopic stabilization and arthroscopic non-anatomical CC ligament reconstruction were found to reliably provide good clinical outcomes for type III ACJ injuries, although none of the studied techniques demonstrated reliability in maintaining anatomical ACJ reduction after surgery. 


\section{Acknowledgements}

The Authors would like to express their thanks and gratitude towards the following people who supported the study: Eugenia Cucciol, Alessio De Biasi, Domenico Fazio, Tiziano Panighel, Mara Buoro, Alessandra Prosdocimo, Nadia Tonetto, Antonella Bonora, Lina Fiorin, Valeria Martin of the Oderzo Hospital Orthopaedic and Traumatology Department, Fabio Bucciol, Michela Centenaro, Luca Vernier of the Oderzo Hospital Radiology Department, Margherita Pierantoni of the Rizzoli Orthopaedic Institute, Bologna, Italy.

\section{References}

1. Rockwood CA, Young DC. Disorders of the acromioclavicular joint. In: Rockwood CA, Matsen FA (eds) The Shoulder. Philadelphia, Sanders. 1990; Vol 1:413-476.

2. Calvo E, López-Franco M, Arribas IM. Clinical and radiologic outcomes of surgical and conservative treatment of type III acromioclavicular joint injury. J Shoulder Elbow Surg. 2006;15: 300-305

3. Taft TN, Wilson FC, Oglesby JW. Dislocation of the acromioclavicular joint. J Bone Joint Surg Am. 1987;69:10451051.

4. Yoo YS, Tsai AG, Ranawat AS, Bansal M, Fu FH, Rodosky MW, et al. A biomechanical analysis of the native coracoclavicular ligaments and their influence on a new reconstruction using a coracoid tunnel and free tendon graft. Arthroscopy. 2010;26:1153-1161

5. Fukuda K, Craig EV, An KN, Cofield RH, Chao EY. Biomechanical study of the ligamentous system of the acromioclavicular joint. J Bone Joint Surg Am. 1986;68:434-440.

6. Costic RS, Labriola JE, Rodosky MW, Debski RE. Biomechanical rationale for development of anatomical reconstructions of coracoclavicular ligaments after complete acromioclavicular joint dislocations. Am J Sports Med. 2004;32: 1929-1936.

7. Carofino BC, Mazzocca AD. The anatomic coracoclavicular ligament reconstruction: surgical technique and indications. J Shoulder Elbow Surg. 2010;19 (2 Suppl):37-46.

8. Fraschini G, Ciampi P, Scotti C, Ballis R, Peretti GM. Surgical treatment of chronic acromioclavicular dislocation: comparison between two surgical procedures for anatomic reconstruction. Injury. 2010;41:1103-1106.

9. Giannotti S, Dell'osso G, Bugelli G, Cazzella N, Guido G. Surgical treatment of acromioclavicular dislocation with LARS artificial ligament. Eur J Orthop Surg Traumatol. 2013;23:873-876.

10. Lu N, Zhu L, Ye T, Chen A, Jiang X, Zhang Z, et al. Evaluation of the coracoclavicular reconstruction using LARS artificial ligament in acute acromioclavicular joint dislocation. Knee Surg Sports Traumatol Arthrosc. 2014,22: 2223-2227.

11. Salzmann GM, Walz L, Buchmann S, Glabgly P, Venjakob A, Imhoff AB. Arthroscopically assisted 2-bundle anatomical reduction of acute acromioclavicular joint separations. Am J Sports Med. 2010;38:1179-1187.
12. Zooker CC, Parks BG, White KL, Hinton RY. TightRope versus fiber mesh tape augmentation of acromioclavicular joint reconstruction: a biomechanical study. Am J Sports Med. 2010;38:1204-1208.

13. Andreani L, Bonicoli E, Parchi P, Piolanti N, Michele L. Acromio-clavicular repair using two different techniques. Eur J Orthop Surg Traumatol. 2014;24:237-242.

14. DeBerardino TM, Pensak MJ, Ferreira J, Mazzocca AD. Arthroscopic stabilization of acromioclavicular joint dislocation using the AC graftrope system. J Shoulder Elbow Surg. 2010;19 (2 Suppl):47-52.

15. Padulo J, Oliva F, Frizziero A, Maffulli N. Muscles, Ligaments and Tendons Journal. Basic principles and recommendations in clinical and field science research. Muscles Ligaments Tendons J. 2014;3:250-252.

16. Hudak PL, Amadio PC, Bombardier C. Development of an upper extremity outcome measure: the DASH (disabilities of the arm, shoulder and hand) [corrected]. The Upper Extremity Collaborative Group (UECG). Am J Ind Med. 1996;29:602-608.

17. Charles E, Kumar V, Blacknall J, Edwards K, Geoghegan JM, Manning PA, et al. A validation of the Nottingham Clavicle Score: a clavicle, acromio-clavicular joint and sterno-clavicular joint specific patient reported outcome measure. Bone Joint J. 2013;95-B (Suppl 1):50.

18. Constant CR, Murley AH. A clinical method of functional assessment of the shoulder. Clin Orthop Relat Res. 1987; (214):160-164.

19. Bearden JM, Hughston JC, Whatley GS. Acromioclavicular dislocation: method of treatment. J Sports Med. 1973;1:5-17.

20. Tauber M, Gordon K, Koller H, Fox M, Resch H. Semitendinosus tendon graft versus a modified Weaver-Dunn procedure for acromioclavicular joint reconstruction in chronic cases: a prospective comparative study. Am J Sports Med. 2009;37:181-190.

21. Boström Windhamre HA, von Heideken JP, Une-Larsson VE, Ekelund AL. Surgical treatment of chronic acromioclavicular dislocations: a comparative study of Weaver-Dunn augmented with PDS-braid or hook plate. J Shoulder Elbow Surg. 2010;19:1040-1048.

22. Fraser-Moodie JA, Shortt NL, Robinson CM. Injuries to the acromioclavicular joint. J Bone Joint Surg Br. 2008;90:697707.

23. Beitzel K, Cote MP, Apostolakos J, Solovyova O, Judson CH, Ziegler CG, et al. Current concepts in the treatment of acromioclavicular joint dislocations. Arthroscopy. 2013;29:387397.

24. Walz L, Salzmann GM, Fabbro T, Eichhorn S, Imhoff AB. The anatomic reconstruction of acromioclavicular joint dislocations using 2 TightRope devices: a biomechanical study. Am J Sports Med. 2008;36:2398-2406.

25. Harris RI, Wallace AL, Harper GD, Goldberg JA, Sonnabend $\mathrm{DH}$, Walsh WR. Structural properties of the intact and the reconstructed coracoclavicular ligament complex. Am J Sports Med. 2000;28:103-108.

26. Scheibel M, Dröschel S, Gerhardt C, Kraus N. Arthroscopically assisted stabilization of acute high-grade acromioclavicular joint separations. Am J Sports Med. 2011;39: $1507-1516$. 